\title{
Biochemical mechanisms of cataract in diabetes mellitus
}

\author{
Daniela MIRICESCU ${ }^{1}$, Maria GREABU ${ }^{1}$, Constantin STEFANI ${ }^{2,3}$, lulia-loana STANESCU ${ }^{4}$, \\ Ana Maria Alexandra STANESCU ${ }^{2}$, Alexandra TOTAN ${ }^{1}$ \\ ${ }^{1}$ Department of Biochemistry, Faculty of Dental Medicine, "Carol Davila" University of Medicine \\ and Pharmacy, Bucharest, Romania \\ 2Department of Family Medicine, "Carol Davila" University of Medicine and Pharmacy, \\ Bucharest, Romania \\ 3"Carol Davila" University Central Emergency Military Hospital, Bucharest, Romania \\ "Department of Physiology, Faculty of Dental Medicine, "Carol Davila" University of Medicine and \\ Pharmacy, Bucharest, Romania
}

\section{ABSTRACT}

Cataract is one of the most common diabetes mellitus complication among the adult population worldwide. The purpose of this review is to describe how reactive oxigen species (ROS) are produced, the antioxidant enzymes involved in their neutralization, and the molecular mechanisms associated with hyperglycemia that contributes to cataract development.
\end{abstract}

Keywords: diabetes mellitus, cataract, biochemical mechanisms

\section{INTRODUCTION}

In the 1990s, the incidence of type 1 diabetes in children varies between 3.4 and $6.3 \%$ depending on the country, with an increased incidence in children under 5 years [1]. In 2012, the results of studies conducted over a period of 20 years (19891998 and 1999-2008) regarding the incidence of type 1 diabetes in children under 15 years from 19 countries were published. During this time, the countries included in the study reported an increase in the incidence of type 1 diabetes in children of 3.3-3.4\% [2]. In Europe, the incidence of diabetes among the adult population is $8.6 \%$, with an incidence of $2.4 \%$ in Moldova and $14.9 \%$ in Turkey. By 2035, an increase of diabetic mellitus incidence is expected in children but also in adults
[3]. Diabetic patients, present many systemic complications and ocular damages are common disorders. Worldwide, cataracts are the leading cause $(90 \%)$ of visual impairment. The aging of the European population is associated with an increased incidence of cataracts. Thus, in people over 70 years, a doubling of cataract incidence is expected from $10.5 \%$ reported in 2009 to $20.4 \%$ in 2050.

Cataract is a multifactorial disorder, in its'pathogenesis being involved several factors:

- diabetes mellitus

- age

- sex (especially women)

- genetic predisposition

- drug administration (prednison)

- exposure to UV radiation 
- smoking

- alcohol

- blood pressure / hypertension

- education level [4]

\section{REACTIVE OXIGEN SPECIES (ROS) GENERATION}

The results of the clinical and experimental studies performed to date highlight the involvement of oxidative stress (OS) in the initiation and progression of many systemic and oral disorders [5-7]. ROS are produced as intermediates and by-products in the mitochondrial respiratory chain and in the endoplasmic reticulum (ER). Neutrophils and macrophages generate ROS, during an inflammatory process but also in other normal metabolic reactions. In addition to the internal sources of ROS, there are a number of external factors that lead to the formation of ROS, UV radiation, cigarette smoke, alcohol and drugs [5,8-10].

Prolonged exposure to oxidizing agents, heavy metal ions, herbicides, pesticides, ultraviolet, ionizing radiation, together with ROS from normal physiological processes, contributes to DNA, lipid and protein damage. At the cellular level, if DNA is unrepairable, to avoid the propagation of the mutations produced by ROS, apoptosis is induced, as a mechanism of DNA repair. The cells are protected against the harmful effects of ROS, by antioxidant enzymes (superoxidismutase-SOD, catalase-CAT, glutathione peroxidase-GPx), glutathione (GSH) and antioxidant vitamins (vitamins C, E and A) [5].

In the first stage of ROS formation, $\mathrm{O}_{2}$ is reduced to superoxide anion $\mathrm{O}_{2}^{-}$, a reaction that is carried out in phagocytosis by enzyme NADPH oxidase, NADH dehydrogenase (complex I) and coenzyme Q (complex III). GSH and SOD neutralizes $\mathrm{O}_{2}$ SOD deficiency is associated with mutagenesis process, and other pathologies such as lactic acidosis, cardiomyopathy, neurodegenerative disorders, muscle atrophy, fertility disorders [8-10].

$$
\begin{gathered}
\mathrm{O}_{2}+1 e^{-}=\mathrm{O}_{2}^{-} \\
2 \mathrm{O}_{2}^{-}+2 \mathrm{GSH}+2 \mathrm{H}^{+} \rightarrow 2 \mathrm{H}_{2} \mathrm{O}_{2}+\mathrm{GSSG} \\
\mathrm{H}_{2} \mathrm{O}_{2}+2 \mathrm{GSH} \rightarrow 2 \mathrm{H}_{2} \mathrm{O}+\mathrm{GSSG} \\
4 \mathrm{O}_{2}^{--}+2 \mathrm{H}_{2} \mathrm{O} \stackrel{\text { SOD }}{\longrightarrow} 3 \mathrm{O}_{2}+4 \mathrm{HO}^{-} \\
2 \mathrm{O}_{2}^{-}+2 \mathrm{H}^{+} \stackrel{\text { SOD }}{\longrightarrow} \mathrm{H}_{2} \mathrm{O}_{2}
\end{gathered}
$$

FIGURE 1. ROS formation and SOD neutralization [adapted from 11]

Hydrogen peroxide $\left(\mathrm{H}_{2} \mathrm{O}_{2}\right)$ is also formed at peroxisomes level, by the reduction reaction of $\mathrm{O}_{2}$ with fatty acids, polyamides, catalyzed by FAD (flavin adenindinucleotide). This harmful molecule of the body is also produced by degradation of AMP (adenosine monophosphate) with formation of hypoxanthine (reaction catalyzed by xanthine oxidase-XO), which is further oxidized to xanthine and in the end to uric acid [11].

$$
\begin{aligned}
& \mathrm{Hypoxanthine}+\mathrm{H}_{2} \mathrm{O}+\mathrm{O}_{2} \stackrel{\text { Xo }}{\rightarrow} \text { Xanthine }+ \\
& \mathrm{H}_{2} \mathrm{O}_{2} \stackrel{\text { XO }}{\rightarrow} \text { Uric acid }
\end{aligned}
$$

According to the following reaction, in peroxisomes, catalase uses $\mathrm{H}_{2} \mathrm{O}_{2}$, to oxidize alcohols, phenols, ethanal, methanal and formic acid:

$$
\mathrm{R}-\mathrm{OH}+\mathrm{H}_{2} \mathrm{O}_{2} \stackrel{\mathrm{CAT}}{\longrightarrow} \mathrm{R}+2 \mathrm{H}_{2} \mathrm{O}
$$

Excess $\mathrm{H}_{2} \mathrm{O}_{2}$ is converted by CAT to $\mathrm{O}_{2}$ and $\mathrm{H}_{2} \mathrm{O}$ as follows:

$$
\mathrm{H}_{2} \mathrm{O}_{2} \text { (excess) } \stackrel{\mathrm{CAT}}{\longrightarrow} 1 / 2 \mathrm{o}_{2}
$$

The Fenton reaction generates the most dangerous ROS, the hydroxyl radical $(\mathrm{HO})$, which has the ability to damage DNA.

$$
\mathrm{H}_{2} \mathrm{O}_{2}+\mathrm{Fe}^{2+}\left(\mathrm{Cu}^{+}\right) \stackrel{\text { Fenton reaction }}{\longrightarrow} \mathrm{HO}+\mathrm{Fe}^{3+}\left(\mathrm{Cu}^{2+}\right)
$$

\section{HYPERGLICEMIA AND ROS}

Hyperglycemia leads to overproduction of $\mathrm{O}_{2}^{-}$ by four molecular mechanisms:

- polyol pathway

- advanced glycation end products (AGEs) formation

- activation of protein kinase C (PKC)

- hexosamine pathway

\section{Polyol pathway}

$$
\begin{aligned}
& \uparrow \text { Fructose }-6-P \stackrel{\text { GFAT }(G l n \rightarrow G l u)}{\longrightarrow} \uparrow \text { Glucosamine }-6- \\
& P \rightarrow \uparrow U D P-G l c N A c
\end{aligned}
$$

A central role in cataract development plays the polyol pathway via the enzyme aldolase reductase (ER), which catalyzes glucose reduction into sorbitol. The intracellular accumulation of sorbitol generates a hyperosmotic effect, with the formation of hydropic fibers of the lens that degenerate and lead to cataract formation. In diabetic patients, the production of sorbitol takes place more quickly compared to nondiabetic patients, which is then converted under the action of sorbitol dehydrogenase to fructose. Studies performed on laboratory animals have reported that intracellular accumulation of polyols generates liquefaction of lens, leading to opacities of the lens $[12,13]$.

The osmotic stress that results from the accumulation of sorbitol, induces stress in the endoplasmic reticulum (ER), the main site of protein synthesis, with the generation of free radicals. Hyperglycemia causes stress at the ER level, gen- 
erating lens fiber damage. Osmotic stress induced by sorbitol accumulation in the lens, induces apoptosis of the lens epithelial cells and cataracts formation [14-16].

\section{Hexosamine pathway}

$\uparrow$ GA-3-P $\rightarrow \uparrow$ DHAP + NADH $\rightarrow \uparrow \alpha$-glycerol-P $\rightarrow \uparrow D A G \rightarrow \uparrow P K C$

Excess plasma glucose leads to $\mathrm{NADH}, \mathrm{H}^{+}$formation (tricarboxylic acid cycle), a single proton can be transferred by $\mathrm{NADH}, \mathrm{H}^{+}$to $\mathrm{O}_{2}$ with $\mathrm{O}_{2}^{-}$generation. $\mathrm{O}_{2}^{-}$partially inhibits a key enzyme from glycolysis, glyceraldehyde- 3-phosphate dehydrogenase (GADPH), glucose and glycolytic intermediates spill into the polyol and hexosamine pathways.Glutamine-fructose-6-phosphateaminotransferase (GFAT) catalyzes the conversion of glutamine into glucose and glucosamine-6-phosphate formation. Increased flux of fructose-6-phosphate (fructose-6-P) leads to increased levels of glucoamine-6-P and UDP-N-acetylglucosamine (UDP-GlcNAc). The hexosamine pathway mediates increased transcription of inflammatory cytokine-specific genes [16].

\section{Protein kinase $\mathbf{C}$ pathway}

个GA-3-P $\rightarrow$ 个DHAP +

$\mathrm{NADH} \rightarrow \uparrow \alpha$-glycerol- $\mathrm{P} \rightarrow \uparrow \mathrm{DAG} \rightarrow \uparrow \mathrm{PKC}$

$\mathrm{O}_{2}$ - partially inhibits GADPH enzyme, glyceraldehyde-3-phosphate (GA-3-P) is no longer converted to 1,3-diphosphoglycerate and will be isomerized to dihydroxyacetone phosphate (DHAP). DHAP is reduced by NADH to diacylglycerol (DAG) which is an activator of PKC. Once this protein kinase is activated, all three isoforms are also activated: PKC- $\beta$, increases VEGF (vascular endothelial growth factor), PAI-1 (plasminogen activator inhibitor-1) NADPH (nicotinamide adenine dinnucleotide phosphate) and decreases eNOS (endothelial nitric oxide synthase) expressions, which will trigger the angiogenesis process. PKC- $\delta$ activates the MAPK (mitogen-activated protein kinases) signaling pathway with the release of NF-kB (nuclear factor-kB), ROS, PDGFR- $\beta$ (platelet-derived growth factor receptor- $\beta$ ) which will induce cellular apoptosis. PKC- $\zeta$ causes increased expression of NF-kB, TNF- $\alpha$ (tumor necrosis factor), VEGF (vascular endothelial growth factor), thrombin, protein degeneration and retinal blood barrier damage. Activation of PKC isoforms will induce diabetic retinopathy development $[16,17]$.

\section{AGEs pathway}

个GA3P $\rightarrow$ 个methylglyoxal $\rightarrow$ AGEs

Hyperglycemia accelerates the non-enzymatic reactions that take place between the free amino groups of proteins and the carbonyl groups of the reducing carbohydrates such as glucose forming AGEs, through a complex molecular process. AGEs can also be formed from dicarbonyl compounds by auto-oxidation or by degradation of glucose products such as glyoxal, methylglyoxal, glyceradehide. In chronic hyperglycemia, AGEs are actively produced and accumulated in the systemic circulation and different tissues, leading to vascular complications. Increased glucose levels in aqueous humor can lead to glycation of lens proteins, a process that generates increased levels of AGEs $[16,18]$.

AGEs formed by lipids, nucleic acids and proteins, bind to specific cell surface receptors and lead to ROS generation. AGEs activate NF-kB, which will activate transcription of vascular cell adhesion molecule-1 (VCAM-1) and intracellular adhesion molecule-1 (ICAM-1) and ROS generation [19].

\section{OCULAR ANTIOXIDANT SYSTEM}

The eyes are protected from the OS effects by a powerful antioxidant system that includes the SOD enzyme. Three isofromes of SOD are known; the copper / zinc dependent -cytosolic form ( $\mathrm{Cu}$ / Zn-SOD or SOD1); manganese-dependent mitochondrial form (Mn-SOD or SOD2) and copper / zinc-dependent extracellular form (EC-SOD or SOD3). These isoforms catalyze the conversion of $\mathrm{O}_{2}^{-}$to $\mathrm{H}_{2} \mathrm{O}_{2}$, which is further reduced to water by GPX or CAT. Cataract development is associated with increased OS and decreased antioxidants with age. The proteins found in the lens are crosslinked by the disulfide bonds forming insoluble aggregates that will affect the transparency of the lens. Increased serum levels of malondialdehyde, thiobarbituric acid reactive substances, decreased SOD levels, suggest the existence of OS in diabetic patients and lens degeneration $[20,21]$.

\section{CONCLUSIONS}

Diabetes mellitus is associated with many systemic complications, ocular impairment being quite common among adult patients. Cataract is a multifactorial disorder, diabetes mellitus playing an central role in its pathogenesis. Four molecular mechanisms are involved in cataract development leading to the generation of ROS, in particular $\mathrm{O}_{2}^{--}$. The polyol pathway leads to the accumulation of sorbitol, which causes the installation of osmotic stress and damage to the lens fibers. The hexozamine pathway mediates the production of 
proinflammatory cytokines. Through glycolytic intermediates (GA-3-P), PKC signaling pathway is activated and induce ROS generation. Chronic hyperglycemia causes the non-enzymatic reactions with the formation of AGEs, and glycation of proteins from the lens.

\section{Acknowledgement}

All authors equally contributed to the present paper.

\section{Conflict of interest: none declared} Financial support: none declared

\section{REFERENCES}

1. EURODIAB ACE Study Group. Variation and trends in incidence of childhood diabetes in Europe. The Lancet 2000; 355:873-76.

2. Patterson CC, E. Gyürüs E, Rosenbauer J et al. Trends in childhood type 1 diabetes incidence in Europe during 1989-2008: evidence of non-uniformity over time in rates of increase. Diabetologia 2012; 55:2142-47.

3. Tamayo T, Rosenbauer J, Wild SH et al. Diabetes in Europe: An update. Diabetes Res Clin Pract 2014;103(2):206-17.

4. Prokofyeva E, Wegener A, Zrenner E. Cataract prevalence and prevention in Europe: A literature review. Acta Ophthalmol 2012;1755-3768.

5. Valko M, Leibfritz D, Moncol J et al. Free radicals and antioxidants in normal physiological functions and human disease. Int J Biochem Cell Biol 2007; 39:44-84.

6. Miricescu D, Totan A, Calenic B et al. Salivary biomarkers: Relationship between oxidative stress. Acta Odontol Scand 2014;72(1),42-7.

7. Totan A, Miricescu D, Parlatescu I et al. Possible salivary and serum biomarkers for oral lichen planus. Biotech Histochem 2015;90(7):552-8.

8. Alfadda AA, Sallam RMJ. Reactive oxygen species in health and disease. Biomed Biotechnol 2012;2012:936486.

9. Kraaij MD, Savage NDL, Kooij SW et al. Induction of regulatory $T$ cells by macrophages is dependent on production of reactive oxygen species. Proc Natl Acad Sci USA 2010;107:17686-191.

10. Tobore TO. Towards a comprehensive theory of obesity and a healthy diet: The causal role of oxidative stress in food addiction and obesity. Behav Brain Res 2020;17;384:112560.

11. Hepel M, Andreescu S. Oxidative Stress and Human Health. American Chemical Society 2015; ACS Symposium Series; American Chemical Society: Washington, DC.

12. Kador PF, Wyman M, Oates PJ. Aldose reductase, ocular diabetic complications and the development of topical Kinostat. Prog Retin Eye Res 2016; 54:1-29.

13. Snow A, Shieh B, Chang KC et al. Aldose reductase expression as a risk factor for cataract. Chem Biol Interact 2015; 234 : 247-53.

14. Mulhern ML, Madson CJ, Kador PF et al. Cellular osmolytes reduce lens epithelial cell death and alleviate cataract formation in galactosemic rats. Mol Vis 2007; 13:1397-1405.

15. Li WC, Kuszak JR, Dunn K et al. Lens epithelial cell apoptosis appears to be a common cellular basis for non-congenital cataract development in humans and animals. J Cell Biol 1995; 130: 169-181.

16. Ceriello A, Testa R. Antioxidant antiinflammatory treatment in type 2 diabetes. Diabetes Care 2009; 32(2):232-36.

17. Cheng L, Xiao M, Fengsheng $L$ et al. Oxidative Stress-Related Mechanisms and
Antioxidant Therapy in Diabetic Retinopathy. Oxidative Medicine and Cellular Longevity 2017;ID 9702820, 15 pages.

18. Chawla D, Tripathi AK. Role of advanced glycation end products (AGEs) and its receptor (RAGE)-mediated diabetic vascular complications. Integr Food Nutr Metab 2019; 6:1-6.

19. Vincent HK, Taylor AG. Biomarkers and potential mechanisms of obesity-induced oxidant stress in humans. Int $\mathrm{J}$ Obesity 2006; 30:400-18.

20. Sankaranarayanan R, Abhay RV, Mamidipudi RP. Exploration of Molecular Factors Impairing Superoxide Dismutase Isoforms Activity in Human Senile Cataractous Lenses. Invest Ophthalmol Vis Sci 2013; 54(9): 6224-33.

21. Ozmen B, Ozmen D, Erkin E et al. Lens superoxide dismutase and catalase activities in diabetic cataract. Clin Biochem 2002;35(1):69-72.

22. Ergan E, Ozturk F, Beyazyildiz E et al. Oxidant/antioxidant balance in the aqueous humor of patients with glaucoma. Int $J$ Ophthalmol 2016; 9(2): 249-252.

23. Kaur J, Kukreja S, Kaur A et al. The Oxidative Stress in Cataract Patients. J Clin Diagn Res 2012; 6(10): 1629-32. 